04

\title{
Исследование температурного поля газа в следе импульсного электрического разряда
}

\author{
(C) В.А. Лашков, ${ }^{1}$ Ю.В. Добров, ${ }^{1}$ М.Е. Ренев, ${ }^{1}$ И.Ч. Машек, ${ }^{1}$ Н.Ж. Джайчибеков, ${ }^{2}$ Б.С. Шалабаева ${ }^{2}$ \\ ${ }^{1}$ Санкт-Петербургский государственный университет, \\ 199034 Санкт-Петербург, Россия \\ ${ }^{2}$ Евразийский национальный университет им. Л.Н. Гумилева, \\ 010000 Нур-Султан, Казахстан \\ e-mail: youdobrov@gmail.com
}

Поступило в Редакцию 19 ноября 2021 г.

В окончательной редакции 14 декабря 2021 г.

Принято к публикации 24 декабря 2021 г.

Исследован нагрев газа в следе электрического межэлектродного разряда. Динамика локального нагрева изучена с помощью численных методов и экспериментально с использованием интерферометра. Результаты исследований позволяют оценить изменение распределения температуры в поперечном сечении следа разряда, динамику максимальной температуры нагрева и размеров нагретой области.

Ключевые слова: импульсный межэлектродный разряд, низкотемпературная плазма, нагрев газа.

DOI: 10.21883/JTF.2022.04.52241.294-21

\section{Введение}

Активное управление сверхзвуковыми потоками газа при помощи локального вложения энергии является важной и перспективной задачей современной плазменной аэродинамики. Локальное энерговложение в сверхзвуковой поток газа позволяет воздействовать на характер обтекания различных тел [1-6]: уменьшать коэффициент лобового сопротивления; увеличивать коэффициент подъемной силы, тем самым увеличивая аэродинамическое качество; совершенствовать управление летательным аппаратом; проводить интенсификацию перемешивания, смену режима течения в пограничном слое. Вклад энергии в набегающий сверхзвуковой поток может осуществляться при помощи различных видов разрядов [7-11]: лазерная искра, микроволновый разряд, электрический межэлектродный разряд, диэлектрический барьерный разряд.

Важным вопросом при использовании нагретого канала в аэродинамических приложениях является эффективность преобразования подведенной электрической энергии в тепловую энергию газа [6]. Помимо теплового эффекта на структуру течения газа около аэродинамического тела может оказывать влияние неравновесность газа в канале, особенно это проявляется при больших числах Маха набегающего потока [12].

Настоящая работа направлена на исследование термодинамических параметров следа канала, нагретого с помощью электрического межэлектродного разряда, изучение динамики температуры и геометрии. Исследование проводилось экспериментальными методами, результаты которого сравнивались с результатами численного моделирования. В динамике разряда можно выделить две основные фазы: активная, когда к электродам приложено электрическое напряжение, и образуется плазменный канал с неравновесным состоянием газа, и пассивная после отключения напряжения, которая характеризуется переходом газа в канале к равновесному состоянию. В разделе, посвященном активной фазе разряда, определялись геометрия канала и распределение мощности нагрева среды плазмой. Эти данные были использованы при расчете эволюции следа канала в пассивной фазе. Особое внимание уделялось пассивной фазе, так как в аэродинамических приложениях для модификации течения газа часто используется нагретый после разряда след канала. Основная цель исследования установление связи между электрическими параметрами межэлектродного разряда и температурой нагрева газа в следе разряда.

\section{1. Техника экспериментального исследования параметров следа канала}

Разряд создавался в экспериментальной камере путем приложения к электродам импульсного напряжения $20-25 \mathrm{kV}$, длительностью $3 \mu$ s через балластное сопротивление $800 \Omega$. Эксперименты проводились в воздушной среде при атмосферном давлении. Расстояние между электродами типа острие-острие составляло $6 \mathrm{~mm}$. Максимальный ток активной фазы разряда $25 \mathrm{~A}$.

Тепловой след исследуемого разряда по предварительным оценкам вносит очень малые фазовые оптические возмущения в среду (небольшие линейные размеры следа и его малый тепловой нагрев). Двухлучевые интерферометры в этом случае практически неприменимы, поэтому исследование проводилось с помощью многолу- 
чевого внутрикамерного интерферометра Фабри-Перо, описанного в работе [13].

Интерферометр с базой $0.2 \mathrm{~m}$, световым диаметром $0.09 \mathrm{~m}$ освещался через прибор ИАБ-450 одночастотным гелий-неоновым лазером ЛГН-303 с выходной мощностью $1 \mathrm{mV}$, длиной волны $632.8 \mathrm{~nm}$ и относительной девиацией частоты менее $10^{-10}$. Интерференционная картина регистрировалась с помощью быстродействующей интенсифицированной камеры РСО DiCAM-PRO GaAs P46 с минимальным временем экспозиции $5 \mathrm{~ns}$. Обычное время экспозиции в нашем случае составляло порядка $1 \mu \mathrm{s}$. Время задержки экспозиции после начала разряда - от 50 до $1000 \mu \mathrm{s}$.

\section{2. Результаты экспериментального исследования параметров газа в следе разряда}

Значительная концентрация электронов, оставшихся в канале после активной фазы разряда, может существенно влиять на результаты интерферометрических измерений. Однако при атмосферном давлении характерное время релаксации составляет порядка $1 \mu \mathrm{s}$ [14], поэтому при проведении измерений температуры канала на больших временах влиянием электронной составляющей пренебрегалось. Предполагалось, что нагретая разрядом область имеет форму, близкую к осесимметричной. Для получения распределения температуры в поперечном сечении следа разряда использовалась методика, описанная в работе [15].

На рис. 1 в качестве примера представлена интерференционная картина, полученная с помощью интерферометра через $140 \mu$ s после разряда.

Затененные места на рис. 1 - корпус, на котором закреплены электроды, и подводящий электрический кабель. В правом верхнем углу представлен выделенный с помощью графического редактора участок интерференционной картины, который подвергался дальнейшему анализу.

С использованием описанной в [15] методики обработки интерферометрических линий, была получена зависимость изменения температуры $T$ газа в поперечном сечении следа разряда. Результаты измерений показали, что максимальная температура газа на оси следа разряда на $140 \mu$ s составляла $T_{\max }=333 \mathrm{~K}$. Стандартное отклонение при определении температуры не превышало $30 \mathrm{~K}$. Характерный размер теплового следа, оцененный на уровне половины от максимальной температуры, в данном случае составил $S_{5}=2.2 \pm 0.3 \mathrm{~mm}$.

Изменение температуры на оси следа разряда во времени представлено на рис. 2, $a$. На рис. $2, b$ показана временная зависимость нагрева $\alpha=T_{h} / T_{\max }$ канала, где $T_{h}$ - температура окружающего газа. Этот параметр часто используется при компьютерном моделировании взаимодействия нагретого канала с ударным слоем на сверхзвуковом теле $[1,2]$. Исследования показали, что

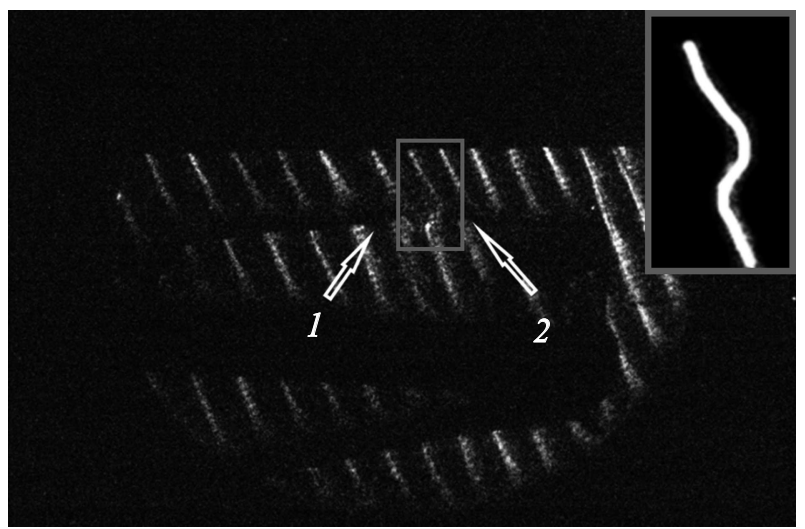

Рис. 1. Общая интерферометрическая картина через $140 \mu \mathrm{s}$ после разряда: 1 - катод, 2 - анод.

параметр нагрева $\alpha$ канала в условиях проведенных экспериментов в промежуток времени 50-1000 $\mu$ s меняется в диапазоне $0.8-0.95$.

К $1000 \mu \mathrm{s}$ температура падает до $310 \mathrm{~K}$. Очевидно, это происходит за счет того, что тепло нагретого канала рассеивается в окружающем пространстве. Это также подтверждается тем, что с увеличением времени после разряда происходит увеличение параметра $S_{50}$ (рис. 3). Эксперименты показали, что параметр $S_{50}$ увеличивается практически линейно. К моменту времени $1000 \mu$ s после разряда параметр $S_{50}$ возрастает примерно до $14 \mathrm{~mm}$.

\section{3. Численное моделирование}

\section{1. Активная фаза разряда}

Модели межэлектродного разряда низкотемпературной плазмы в покоящемся газе представлены в большом количестве работ, например, [14,16-19]. Они используются для решения различных задач: исследование причин ветвления стримера, вычисления параметров ионизированной области, теплового, химического и силового воздействия.

В настоящей работе произведен в $2 D$-осесимметричной постановке самосогласованный численный расчет импульсного межэлектродного разряда в покоящемся воздухе при стандартных условиях. Геометрия расчетной области изображена на рис. 4. Система электродов: две медные иглы. Импульсный источник напряжения $U_{0}(t)$ $(20 \mathrm{kV}$, передний фронт продолжительностью $200 \mathrm{~ns})$ подключен к системе через сопротивление $R=800 \Omega$.

Для расчета параметров разряда использовались следующие уравнения.

Уравнение Пуассона:

$$
-\varepsilon_{0} \Delta \varphi=\rho_{c h}, \rho_{c h}=|e|\left(n^{+}-n_{e}-n^{-}\right),
$$

где $\varepsilon_{0}-$ электрическая постоянная, $\varphi$ - электрический потенциал, $\rho_{c h}-$ плотность электрического заряда, 

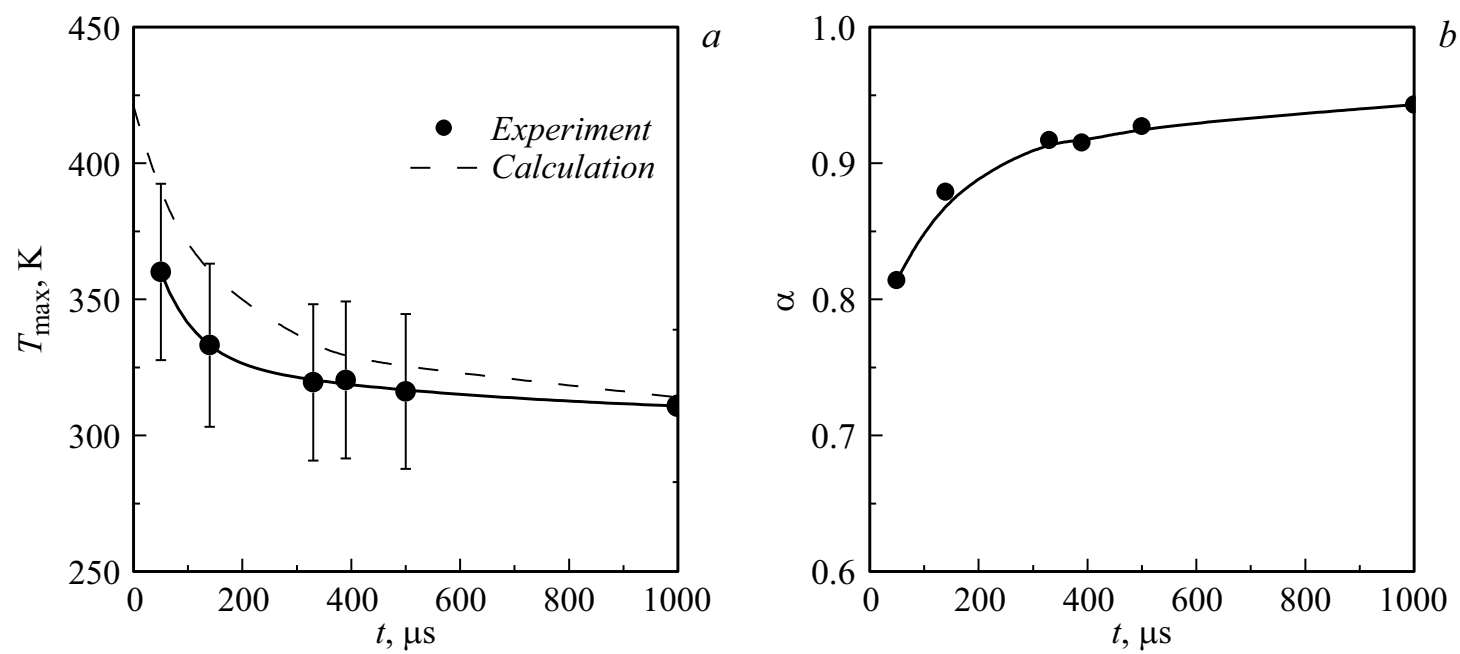

Рис. 2. $a-$ изменение температуры газа на оси нагретого канала $T_{\max }$ от времени с начала разряда, $b-$ зависимость параметра нагрева канала $\alpha$ от времени.

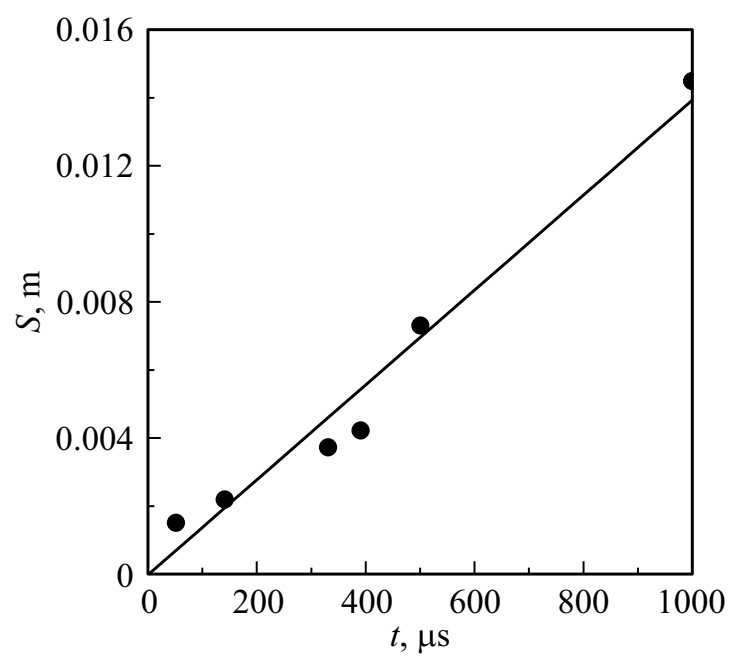

Рис. 3. Расстояние от оси разряда до того места, где температура газа составляет $50 \%$ от максимальной температуры.

$|e|$ - элементарный заряд, $n_{+}, n_{-}, n_{e}-$ концентрации всех положительных, всех отрицательных ионов и электронов, $\mathbf{E}$ - вектор напряженности электрического поля.

Для расчета концентрации электронов использовалось уравнение неразрывности:

$\frac{\partial n_{e}}{\partial t}+\left(\nabla, \mathbf{J}_{e}\right)+\left(\mathbf{V}_{g}, \nabla\right) n_{e}=S_{e}, \mathbf{J}_{e}=-b_{e} \mathbf{E} n_{e}-\nabla D_{e} n_{e}$,

где $\mathbf{J}_{e}-$ вектор потока электронов, $\mathbf{V}_{g}-$ скорость движения среды, $S_{e}-$ источник электронов, $b_{e}, D_{e}-$ коэффициенты подвижности и диффузии электронов.

Уравнение переноса энергии электронов:

$$
\begin{gathered}
\frac{\partial n_{e} \psi}{\partial t}+\left(\nabla, \mathbf{J}_{\psi}\right)+\left(\mathbf{E}, \mathbf{J}_{e}\right)+\left(\mathbf{V}_{g}, \nabla\right) n_{e} \psi=I_{p o t}^{i} S_{i}^{i n}-Q_{e}, \\
\mathbf{J}_{\psi}=-b_{e} \mathbf{E} n_{e} \psi-\nabla D_{e} n_{e} \psi,
\end{gathered}
$$

где $\mathbf{J}_{\psi}-$ вектор потока энергии электронного газа, $\psi$ - средняя энергия электронов, $I_{p o t}^{i}-$ потенциал реакции $i, S_{i}^{i n}-$ скорость реакции неупругих взаимодействий, $Q_{e}-$ мощность нагрева среды за счет упругих столкновений с электронами.

Уравнения Максвелла-Стефана:

$$
\begin{gathered}
\rho_{g}\left(\frac{\partial}{\partial t}+\left(\mathbf{V}_{g}, \nabla\right)\right) \omega_{i}=\left(\nabla, \rho \omega_{i} \mathbf{V}_{i}\right)+S_{i}, \\
\mathbf{V}_{i}=D_{i} \nabla\left(\ln \omega_{i}+\ln M_{n}\right)-z_{i} b_{i} \mathbf{E},
\end{gathered}
$$

где $\rho_{g}-$ плотность среды, $\omega_{i}-$ массовая доля $i$ сорта частиц, $\mathbf{V}_{i}-$ скорость движения частиц $i$-сорта, $S_{i}$ - источник частиц $i$-сорта, $D_{i}, b_{i}, z_{i}$ - коэффициенты диффузии, подвижности в электрическом поле и зарядовое число частиц $i$-сорта, $M_{n}-$ средняя молярная мacca.

Уравнение переноса фотонов и определение скорости реакции фотоионизации [17]:

$$
\frac{c}{-\lambda_{13.6}} \Delta n_{p h-13.6}+\lambda_{13.6} c=S_{N_{2}^{13.6}},
$$

где $c-$ скорость света, $\lambda_{13.6}-$ коэффициент поглощения фотонов чистым кислородом, $n_{p h-13.6}-$ концентрация фотонов с энергией $13.6 \mathrm{eV}, S_{N_{2}^{13.6}}$ - источник

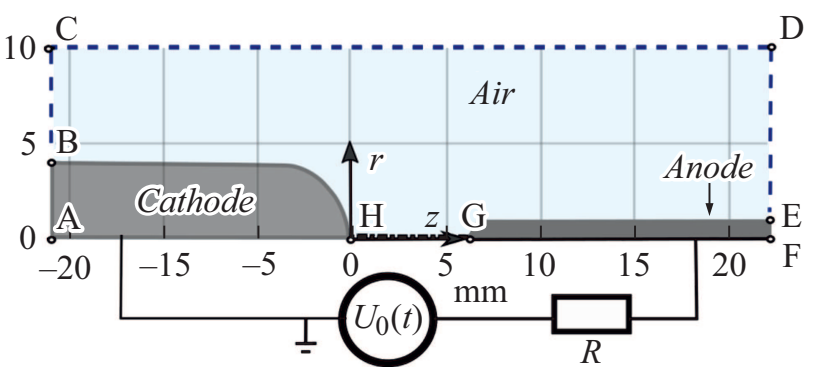

Рис. 4. Геометрия расчетной области. 

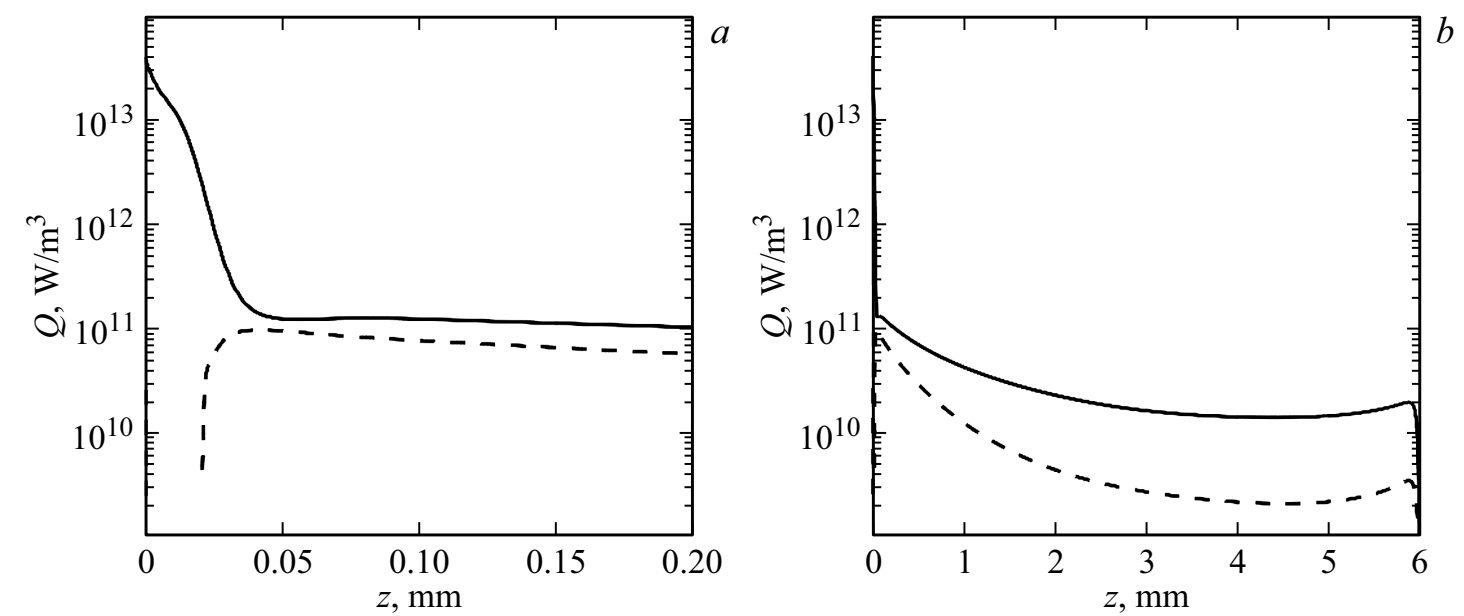

Рис. 5. Распределения мощностей нагрева в момент времени $260 \mathrm{~ns}$ : ионным током (сплошная линия), плазменными реакциями (штриховая линия): $a-$ вблизи катода, $b-$ весь промежуток.

возбужденной $(13.6 \mathrm{eV})$ молекулы азота, $S_{i}^{p h-13.6}-$ фотоионизация.

Уравнения Навье-Стокса и переноса тепловой энергии:

$$
\begin{aligned}
\rho_{g}\left(\frac{\partial}{\partial t}\right. & \left.+\left(\mathbf{V}_{g}, \nabla\right)\right) \mathbf{V}_{g}= \\
& -\nabla p_{g}+\mu_{g}\left(\Delta \mathbf{V}_{g}+\frac{1}{3} \nabla\left(\nabla, \mathbf{V}_{g}\right)\right)+\rho_{c h} \mathbf{E},
\end{aligned}
$$

$\rho_{g} C_{p}\left(\frac{\partial}{\partial t}+\left(\mathbf{V}_{g}, \nabla\right)\right) T_{g}-\nabla\left(k_{g} \nabla T_{g}\right)=Q_{+}+Q_{\text {reac }}+Q_{e}$,

где $p_{g}$ - давление воздуха, $\mu_{g}-$ вязкость газа, $C_{p}-$ удельная теплоемкость среды при постоянном давлении, $T_{g}$ - температура газа, $k_{g}$ - теплопроводность среды, $Q_{+}, Q_{\text {reac }}$ - мощности нагрева среды за счет дрейфового движения ионов и реакций (рекомбинации, релаксации, диссоциации и др.).

В приведенной модели использовались допущения, подробно описанные в работе [20]. Реакции диссоциации и ассоциативной ионизации в воздухе при высокой температуре взяты из работы [21]. Реакции рекомбинации, релаксации описаны в работах [16,22]. Определение мощностей нагрева происходило согласно [16]. В модели рассматривались следующие сорта частиц: $e, \mathrm{O}_{2}, \mathrm{O}_{2}^{+}$, $\mathrm{O}_{2}(\mathrm{a} 1 \mathrm{~d}), \mathrm{O}, \mathrm{O}^{+}, \mathrm{O}^{-}, \mathrm{N}_{2}, \mathrm{~N}_{2}^{+}, \mathrm{N}_{2} \mathrm{a}, \mathrm{N}_{2} \mathrm{~A}, \mathrm{~N}, \mathrm{~N}^{+}, \mathrm{NO}, \mathrm{NO}^{+}$ и фотоны с энергией $13.6 \mathrm{eV}$.

В начальный момент времени между электродами имеется однородная естественная концентрация затравочных электронов и ионов кислорода $10^{13} 1 / \mathrm{m}^{3}$.

Расчет показал, что в заданных условиях разряд зажигается после $250 \mathrm{~ns}$. На рис. 5 представлены рассчитанные к моменту времени $260 \mathrm{~ns}$ пространственные распределения объемных мощностей нагрева ионным током (сплошная линия) и плазменными реакциями (штриховая линия). При расчете в прикатодном слое (его толщина менее $0.05 \mathrm{~mm}$ ) джоулев нагрев плазмы ионным током достигает $5 \cdot 10^{13} \mathrm{~W} / \mathrm{m}^{3}$ (рис. $5, a$ ), вне слоя (рис. 5, $b$ ) - на 3 порядка меньше. Джоулев нагрев вне прикатодного слоя больше, чем плазмохимический нагрев реакциями, в 3-10 раз. Средняя величина мощности общего нагрева в канале с радиусом $1 \mathrm{~mm}$ - около $4.4 \cdot 10^{10} \mathrm{~W} / \mathrm{m}^{3}$. Это значение быстро устанавливается после зажигания разряда и замыкания межэлектродного промежутка. Мощность нагрева упругими столкновениями электронов пренебрежимо мала и не представлена на графиках.

Таким образом, произведенные расчеты начальной стадии межэлектродного разряда высокого напряжения в покоящейся среде показали, что средняя объемная мощность джоулева нагрева в цилиндрическом канале диаметром $2 \mathrm{~mm}$ составляет $4.4 \cdot 10^{10} \mathrm{~W} / \mathrm{m}^{3}$. Это значение было использовано при моделировании газодинамики пассивной фазы разряда.

\section{2. Пассивная фаза разряда}

Для моделирования газодинамики использовались данные из расчета активной фазы разряда. Численное моделирование проводилось в пакете ANSYS FLUENT. Рассматривалась осесимметричная задача в рамках модели совершенного вязкого газа, для описания газовой динамики решалась система уравнений Навье-Стокса:

$$
\begin{gathered}
\frac{\partial \rho}{\partial t}+\nabla(\rho \mathbf{V})=0, \\
\frac{\partial}{\partial t}(\rho \mathbf{V})+\nabla(\rho \mathbf{V V})=-\nabla p+\nabla(\overline{\overline{\boldsymbol{\tau}}}), \\
\frac{\partial}{\partial t}(\rho E)+\nabla(\mathbf{V}(\rho E+p))=\rho q+\nabla(\lambda \nabla T),
\end{gathered}
$$

где $\rho$ - плотность, $\mathbf{V}$ - вектор скорости, $p$ - давление, $\tau$ - тензор напряжений, $E-$ полная энергия, $q-$ источниковый член энергии, $\lambda-$ коэффициент теплопроводности, $T$ - температура. Для замыкания системы 

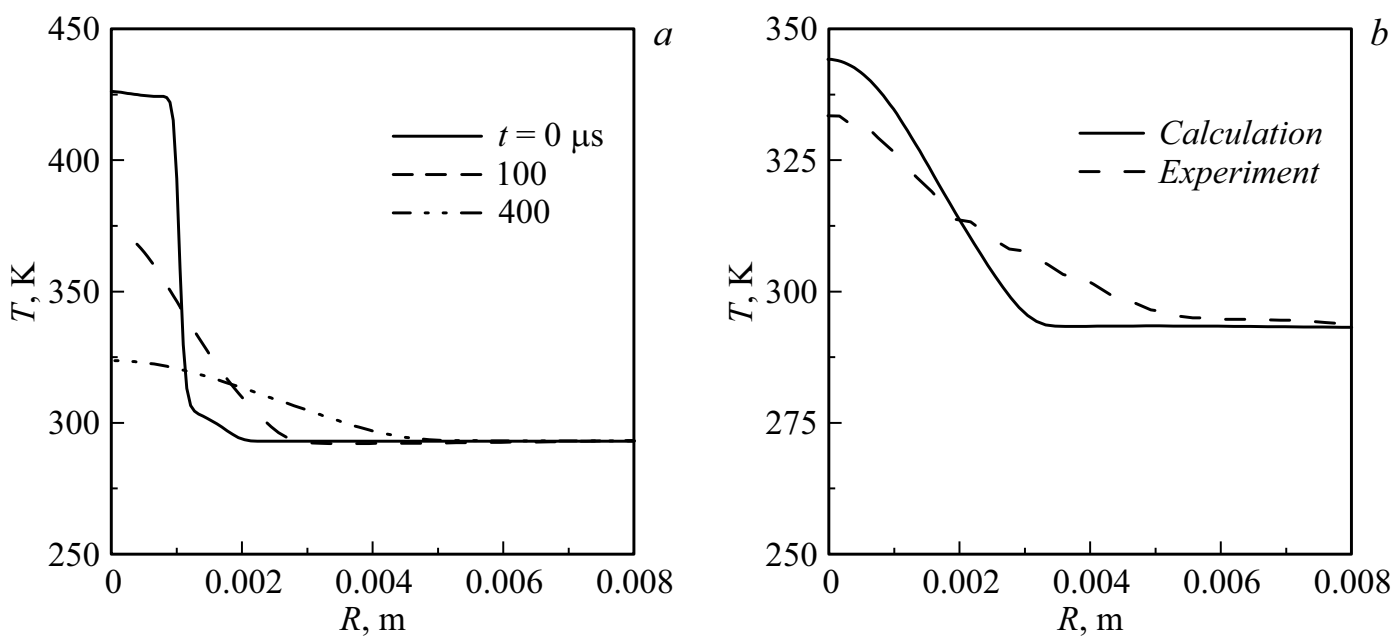

Рис. 6. $a$ - распределение температуры в сечении для разных моментов времени после разряда, $b-$ распределение температуры в сечении через $140 \mu$ s после разряда.

уравнений использовались уравнение состояния идеального газа и $k$-epsilon модель турбулентности.

Задача решалась при следующих начальных условиях: стандартное давление среды, температура - $293 \mathrm{~K}$. Область энерговложения представляла собой цилиндр длиной $6 \mathrm{~mm}$ и диаметром $2 \mathrm{~mm}$. В течение $2.7 \mu \mathrm{s}$ эта область нагревалась с мощностью $4.4 \cdot 10^{10} \mathrm{~W} / \mathrm{m}^{3}$. Величина вкладываемой энергии составляет порядка $2.5 \mathrm{~mJ}$. Подведенная электрическая энергия, измеренная в эксперименте - $22 \mathrm{~mJ}$. Таким образом, эффективность использования электрического разряда для нагрева канала газа составила $11 \%$. На рис. 6, $a$ представлено распределение температуры по радиусу $R$ в центральном сечении нагретого канала для разных моментов времени. На рис. $6, b$ представлено сравнение расчетных и экспериментальных данных для $140 \mu$ s после разряда.

Температура газа на оси канала уменьшается во времени с $427 \mathrm{~K}$ после активной фазы разряда до $373 \mathrm{~K}$ после $100 \mu \mathrm{s}$ (см. штриховую линию на рис. 6, $a$ ). Можно отметить, что в пределах точности исследуемых параметров результаты расчетов удовлетворительно совпадают с данными эксперимента.

\section{Выводы}

Проведено экспериментальное исследование эволюции температуры газа в следе импульсного электрического межэлектродного разряда. С помощью интерферометрического метода получены новые данные по динамике температуры газа. Выполнено численное моделирование активной фазы разряда. По полученным данным из расчета активной фазы произведены расчеты изменения температуры газа в пассивной фазе разряда. Полученные в эксперименте данные удовлетворительно совпадают с результатами вычислений.
В исследуемых условиях расчетная температура газа на оси канала после активной фазы в следе разряда составила $427 \mathrm{~K}$, что соответствует параметру $\alpha=0.68$. С увеличением времени после разряда температура газа в канале достаточно быстро падает: к $140 \mu$ s температура падает примерно на 100 К. Характерные размеры нагретой области увеличиваются линейно с ростом времени и в исследованном диапазоне параметров составляют $14 \mathrm{~mm}$ к $1 \mathrm{~ms}$ после разряда. Эффективность использования энергии импульсного электрического разряда для нагрева газа в следе канала составила порядка $10 \%$.

\section{Благодарности}

Исследования проведены с использованием оборудования ресурсного центра Научного парка СПбГУ „Вычислительный центр“.

\section{Финансирование работы}

Работы выполнены при финансовой поддержке Комитета науки Министерства образования и науки Республики Казахстан. Проект: АР09562291.

Благодарим Санкт-Петербургский государственный университет за финансовую поддержку в рамках проекта Мероприятие 1 (id 84912260).

\section{Конфликт интересов}

Авторы заявляют, что у них нет конфликта интересов.

\section{Список литературы}

[1] П.Ю. Георгиевский, В.А. Левин. Тр. МИАН СССР, 186, 197 (1989).

[2] O.A. Azarova, V.G. Grudnitsky, Yu.F. Kolesnichenko. Мат. моделирование, 18 (1), 79 (2006). 
[3] С.М. Аульченко, В.П. Замураев, А.П. Калинина. Прикладная математика и техническая физика, 50 (5), 36 (2009).

[4] O.A. Azarova, D. Knight, Yu.F. Kolesnichenko. Prog. Flight Phys., 5, 139 (2013). DOI: 10.1051/eucass/201305139

[5] N. Kianvashrad, D. Knight, S.P. Wilkinson, A. Chou, G.B. Beeler, M. Jangda. Effect of Of-Body Laser Discharge on Drag Reduction of Hemisphere Cylinder in Supersonic Flow-Part II. AIAA 2018-1433. AIAA Aerospace Sciences Meeting, Kissimmee, Florida, 8-12 January (2018). DOI: $10.2514 / 6.2018-1433$

[6] V.A. Lashkov, A.G. Karpenko, R.S. Khoronzhuk, I.Ch. Mashek. Phys. Plasmas, 23, 052305 (2016). DOI: $10.1063 / 1.4949524$.

[7] V.A. Lashkov, I.Ch. Mashek, Yu.I. Anisimov, V.I. Ivanov, Yu.F. Kolesnichenko, M.I. Ryvkin, A.A. Gorynya. Gas Dynamic Effect of Microwave Discharge on Supersonic Cone Shaped Bodies. AIAA-2004-671. 42nd AIAA Aerospace Sciences Meeting and Exhibit, Reno, Nevada, 5-8 January (2004). DOI: 10.2514/6.2004-671

[8] P.-Q. Elias, N. Severac, J.-M. Luyssen, J.-P. Tobeli, F. Lambert, etal. Experimental Investigation of Linear Energy Deposition Using Femtosecond Laser Filamentation in a $M=3$ Supersonic Flow. AIAA 2018-4896, Joint Propulsion Conference, Cincinnati, Ohio, 9-11 July (2018). DOI: $10.2514 / 6.2018-48962018$

[9] Ю.В. Добров, В.А. Лашков, И.Ч. Машек, А.В. Митяков, В.Ю. Митяков, С.З. Сапожников, Р.С. Хоронжук. ЖТФ, 91 (2), 240 (2021). DOI: 10.21883/JTF.2021.02.50357.209-20 YYu.V. Dobrov, V.A. Lashkov, I.Ch. Mashek, A.V. Mityakov, V.Yu. Mityakov, S.Z. Sapozhnikov, R.S. Khoronzhuk. Tech. Phys., 66 (2), 229 (2021). DOI: $10.1134 / \mathrm{S} 1063784221020109]$

[10] Y.V. Dobrov, V.A. Lashkov, I.Ch. Mashek, R.S. Khoronzhuk. AIP Conf. Proceedings, 1959, 050009 (2018). DOI: $10.1063 / 1.5034637$

[11] P. Bletzinger, B.N. Ganguly, D. VanWie, A. Garscadden. J. Phys. D: Appl. Phys., 38, R33 (2005). DOI: $10.1088 / 0022-3727 / 38 / 4 / R 01$

[12] О.А. Азарова, А.В. Ерофеев, Т.А. Лапушкина. Письма в ЖТФ, 43 (8), 93 (2017). DOI: $10.21883 /$ PJTF.2017.08.44540.16598

[13] I.Ch. Mashek, Yu.I. Anisimov, V.A. Lashkov, Yu.F. Kolesnichenko. Multibeam Interferometry of SelfSustaining and Laser Induced MW Discharge in air. AIAA 2005-790. 43rd AIAA Aerospace Sciences Meeting and Exhibit, Reno, Nevada, 10-13 January (2005). DOI: $10.2514 / 6.2005-790$

[14] А.В. Самусенко, Ю.К. Стишков. Электрофизические процессы в газах при воздействии сильных электрических полей (ВВМ, СПб., 2012), 649 с.

[15] Г.И. Асеев. Использование интерферометра МахаЦендера для определения пространственного распределения показателя преломления и температуры в пламени (СарГУ, Саратов, 2005)

[16] Ю.П. Райзер. Физика газового разряда (Интеллект, 2009), $691 \mathrm{c}$.

[17] U. Ebert, F. Brau, G. Derks, W. Hundsdorfer, C.-Y. Kao, C. Li, A. Luque, B. Meulenbroek, S. Nijdam, V. Ratushnaya, L. Schäfer, S. Tanveer. Nonlinearity, 24(1), C1 (2011). DOI: $10.1088 / 0951-7715 / 24 / 1 / C 01$

[18] N.St.J. Braithwaite. Plasma Sources Sci. Technol., 9(4), 517 (2000). DOI: 10.1088/0963-0252/9/4/307/meta
[19] K. Kourtzanidis, L.L. Raja, S. Coumar, V. Lago. Numerical Simulation of DC Glow Discharges for Shock Wave Modification. AIAA 2016-2157. 54th AIAA Aerospace Sciences Meeting, San Diego, California, 4-8 January (2016). DOI: $10.2514 / 6.2016-2157$

[20] M.E. Renev, Yu.V. Dobrov, V.A. Lashkov, I.Ch. Mashek. Vestnik St.Petersburg University. Mathematics. Mechanics. Astronomy, 54 (4), 428 (2021). https://doi.org/10.1134/S1063454121040154

[21] C. Park, J.T. Howe, R.L. Jaffe, G.V. Candler. J. Thermophys. Heat Transfer, 8 (1), 9 (1994). DOI: 10.2514/3.496

[22] A.I. Saifutdinov, E.V. Kustova, A.G. Karpenko, V.A. Lashkov. Plasma Phys. Rep., 45 (6), 602 (2019).

DOI: $10.1134 / \mathrm{S} 1063780 \mathrm{X} 19050106$ 